\title{
Self-Ion Assisted Modification of Elastomer and Its Micro- and Macroscopic Properties
}

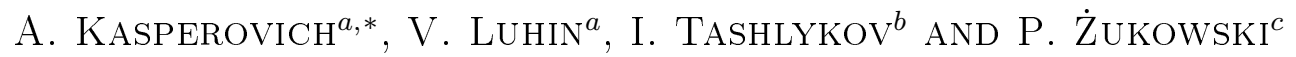 \\ ${ }^{a}$ Department of Technology of Petrochemical Synthesis and Polymer Materials Processing \\ Belarusian State Technology University, 13a, Sverdlova Str., 220006 Minsk, Belarus \\ ${ }^{b}$ Belarusian State Pedagogical University, Minsk, Belarus \\ ${ }^{c}$ Lublin University of Technology, Lublin, Poland
}

\begin{abstract}
The composition of Zr-based thin films on rubber was investigated by utilizing the Rutherford backscattering technique and RUMP code simulation. The level of adhesion between the coating fabricated on rubber by means of self-ion assisted deposition was measured using Pin Pull Test. The coating deposited on the rubber consists of $\mathrm{Zr}, \mathrm{O}, \mathrm{C}, \mathrm{H}$. The self-ion assisted deposition process may successfully control the level of adhesion of the coating to the rubber and causes strong modification of the macroscopic properties of the rubber surface.
\end{abstract}

DOI: 10.12693/APhysPolA.125.1421

PACS: 81.05.Lg, 66.30.Fq, 81.15.-z, 68.35.Np

\section{Introduction}

Wet metallization is still the widely used technique to provide protective or decorative layers. However, because elastomers are often difficult to metallize in solution with good adhesion, and because of environmental considerations there is an increasing need to use the various vacuum methods for deposition of the protective coatings. About one of the successful attempt to apply ion beam technology for metallization from the vapour phase of composite materials is reported in [1]. Deposition of modificative coatings on rubber $(\mathrm{R})$ as one of the elastomers has not been yet studied in detail.

Therefore, we started an investigation of rubber metallization using ion beam technique [2]. In our study coating/rubber structures have been prepared by a method which we called the self-ion-assisted deposition technique (SIAD) [3]. The recent work describes the composition of $\mathrm{Me}$ (Cr, Zr, Mo, Ti, W)-based thin film deposited on rubber with a sulfuric vulcanizing group, the friction properties of the modified rubber and presents some data about the bonding between rubber and the coatings [4]. In this paper we will consider the results of surface modification of rubber with peroxide vulcanizing group by $\mathrm{Zr}^{+}$ions.

\section{Experimental details}

Film deposition was carried out in a vacuum chamber with a base pressure in the range of $10^{-2} \mathrm{~Pa}$ established by the conventional diffusion pump charged with silicon oil. SIAD experiments were performed using a resonance vacuum arc ion source, which provides in correspondence with chosen metal electrodes $(\mathrm{Me}=\mathrm{Zr}$ )

*corresponding author; e-mail: andkasp@belstu.by a mixture of neutral fraction of Me and ionized fraction of Me. A schematic diagram of the SIAD system is given in Fig. 1.

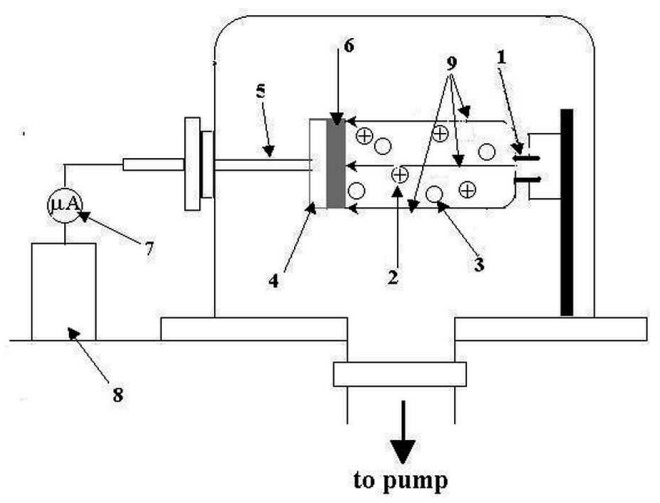

Fig. 1. Schematic representation of SIAD: 1 - vacuum arc ion source, 2 - ionized fraction, 3 - neutral fraction, 4 - target, 5 - target holder, 6 - modified surface, 7 - current integrator, 8 - high voltage power supply, 9 - electric field.

Rubber is insulator, but there were no problems with charging up the insulator because of conductive properties of SIAD coatings, connected with the target holder 5 in Fig. 1.

The relative ratio of ions/neutrals (I/A) could be changed by varying the spacing of the Me source electrodes, by changing the frequency of the source supply, or by varying the target-source separation distance. Substrates were $2 \mathrm{~mm}$ plates of rubber with peroxide vulcanizing group floated to a negative potential with respect to the source in interval $3 \mathrm{kV}$ and $20 \mathrm{kV}$ to accelerate the ion species. The number of ions was estimated from integrated current measurements and the neutrals from measurements of film thickness on unbiased substrate over 
known period of time. From such measurements it was found that $\mathrm{I} / \mathrm{A}$ ratios were between 0.1 and 0.3 and film deposition rates were between $0.01-0.04 \mathrm{~nm} / \mathrm{s}$. The total coating thicknesses typically were $200-700 \mathrm{~nm}$.

The deposited films were analyzed by the Rutherford backscattering spectroscopy (RBS) in conjunction with RUMP code computer simulation of the target content [5]. The energy of $\mathrm{He}^{+}$ions was $2.0 \mathrm{MeV}$, and the scattering, entry and escape angles were 168, 0 and $12^{\circ}$, respectively. The energy resolution of the analyzing system was $25 \mathrm{keV}$.

Topographical properties of the untreated and modified rubber surfaces was investigated by scanning electron microscopy (SEM) and atomic force microscopy (AFM).

A Pin Pull Test (PPT) was employed for measure of the adhesion between the deposited on rubber film and rubber. Five pins were fixed by glue at untreated rubber and modified by SIAD rubber plate. The standard preparation and conditions of the tests were the same as those used in [6].

\section{Result and discussion}

An RBS spectrum of a sample upon which the Zr-based coating was deposited and also irradiated with $\mathrm{Zr}^{+}$ions (Fig. 2, curve 2) is compared to a spectrum of initial rubber (Fig. 2, curve 1). Composition of the Zr-based coating deposited on rubber by means of SIAD presented in Table.

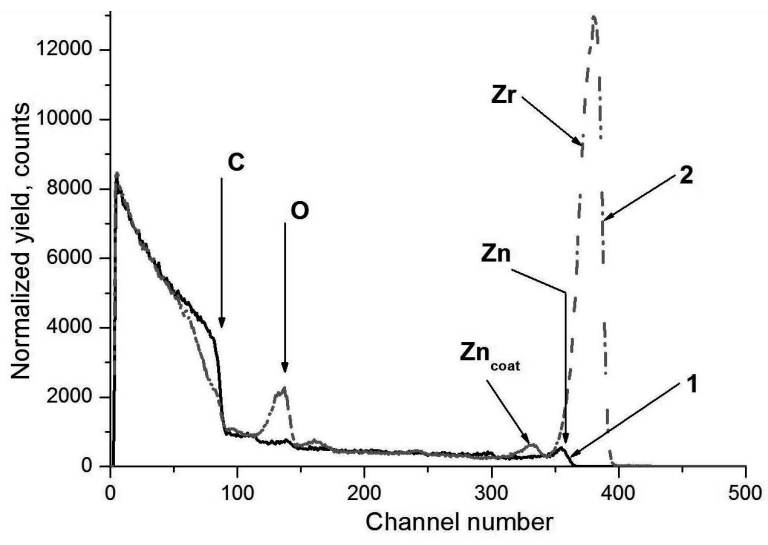

Fig. 2. RBS spectra of untreated rubber - solid curve (1), and with a deposited Zr-based thin film curve (2).

These data indicate that the $\mathrm{Zr}$ concentration decreases from the top surface of the coating to a value about $50 \%$ lower in the middle of the film and increases again near the $\mathrm{Zr} / \mathrm{R}$ interface. The noticeable amount of $\mathrm{Zr}$ is observed in the depth which is $\approx 100 \mathrm{~nm}$ deeper under interface. This means that in the SIAD process the $\mathrm{Zr}$ atoms penetrate deeply into the rubber. The oxygen level follows qualitatively similar trend, which suggests that there is at least some combination of oxygen with $\mathrm{Zr}$ to form an oxide. The carbon and hydrogen
TABLE

Composition of the Zr-based coating deposited on rubber by means of SIAD.

\begin{tabular}{c|c|c|c|c|c}
\hline \hline \multirow{2}{*}{$\begin{array}{c}\text { Depth } \\
{[\mathrm{nm}]}\end{array}$} & \multicolumn{5}{|c}{ Relative content of species [at.\%] } \\
\cline { 2 - 6 } & $\mathrm{Zr}$ & $\mathrm{Zn}$ & $\mathrm{O}$ & $\mathrm{C}$ & $\mathrm{H}$ \\
\hline 15 & - & - & 30.0 & 60.0 & 10.0 \\
165 & 5.20 & - & 17.0 & 9.0 & 71.0 \\
255 & 2.20 & - & 15.0 & 28.0 & 58.0 \\
345 & 2.90 & - & 15.0 & 28.0 & 54.0 \\
495 & 2.60 & - & 14.0 & 28.0 & 55.0 \\
525 & 1.60 & - & 18.0 & 15.0 & 55.0 \\
575 & 4.30 & - & 15.0 & 24.0 & 52.0 \\
605 & 4.50 & - & 25.0 & 50.0 & 18.0 \\
635 & 5.10 & - & 15.0 & 54.0 & 27.0 \\
665 & 0.50 & 0.80 & 10.0 & 56.0 & 29.5 \\
1015 & 0.25 & 0.10 & 3.0 & 30.0 & 61.0 \\
1665 & 0.10 & 0.08 & 2.0 & 30.0 & 42.0 \\
- & 0.08 & 0.05 & 0.04 & 54.9 & 39.0
\end{tabular}

levels are approximately constant in the middle of the coating and are changed near the top surface of the sample and near the coating/ $R$ interface. This high $\mathrm{O}, \mathrm{C}$, and $\mathrm{H}$ content arises, we suppose, because of the used vacuum quality which allows deposition of oxyhydrocarbons onto the growing film and accumulation of them under the irradiation with the accelerated $\mathrm{Me}^{+}$ions. We should emphasise that presence of hydrogen in the SIAD coatings was confirmed quantitatively in the independent study by means of the sharp resonance ${ }^{1} \mathrm{H}\left({ }^{15} \mathrm{~N}, \alpha \gamma\right){ }^{12} \mathrm{C}$ nuclear reaction [7].

The surface of initial rubber shows no periodical or aligned topography. However, after the surface modification by means of SIAD a complicated modified structure can be observed when examined in the SEM, as shown in Fig. 3.

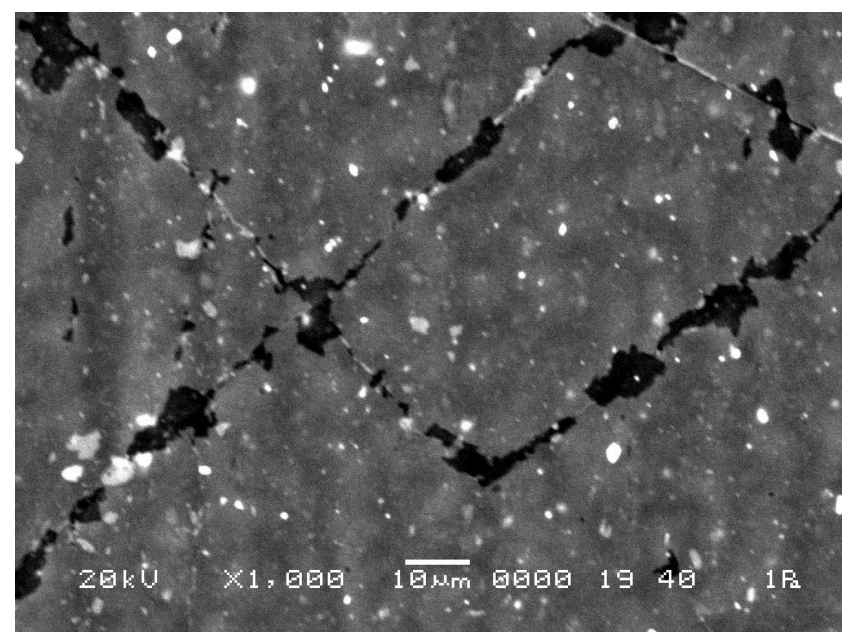

Fig. 3. SEM image of rubber surface modified by Zr. $E_{\mathrm{Zr}+}=10 \mathrm{keV}$. 
The presence of topography on layers produced by ion-assisted deposition is known [4] and was explained quantitatively on a basis of build up in. In the case of the modification of the rubber surface by SIAD "wrinkling" of the deposited layers can be significantly promote by the nonrigid nature of a substrate.

As can be seen from Fig. 4, the surface topography of untreated and modified rubber characterized or "wavy" surface with different heights of protrusions or sharp-edged surface pyramids.

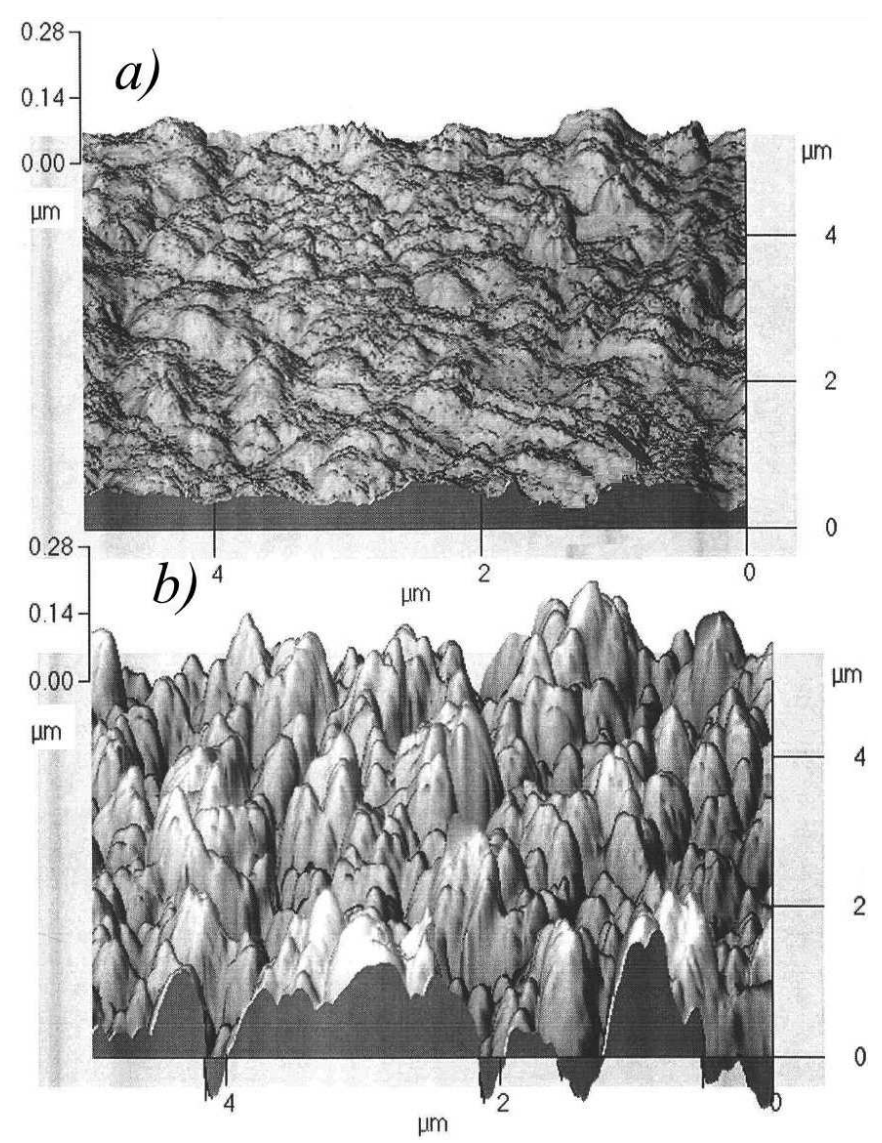

Fig. 4. The surface topography of the raw (a) and modified (b) rubber surface.

This change in surface topography can influence change mechanism of wetting surface various liquid media compared with the original rubber.

The knowledge of the properties of the $\mathrm{Me} /$ elastomer interface is important, particularly to enhance the control of the range of the adhesion. It is found that the formation of metal atoms complexes with the oxygen at the interface promotes a subsequent increase in the adhesion [8]. Previously in [9] we considered that the adhesion between the rubber and the base depends on both the composition of the elastomer and the material of the base. The maximum possible level of adhesion is necessary in metallization of elastomers that are commonly used for the electronic devices, in the packing industry. But sometimes it is more important to achieve a consis- tent adhesion in a more modest level, for example, in a process of the moulding of the rubber articles.

For measuring of the level of the coating adhesion PPT method was applied. The data of our measurements are shown in Fig. 5.

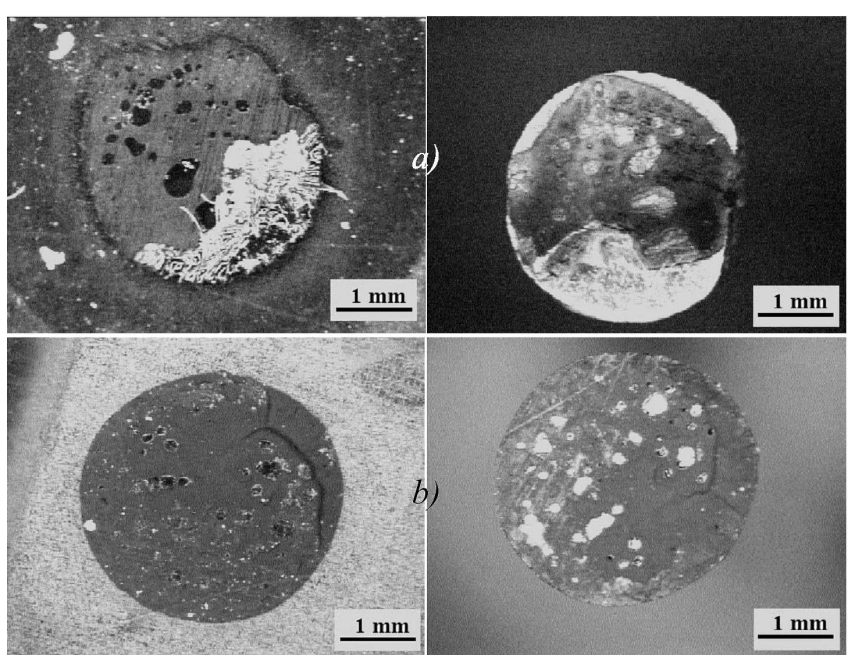

Fig. 5. Images of the sample surface rubber separation (left) and in the corresponding punch (right): (a) original tire, (b) with a coating based on $\mathrm{Zr}$.

The surface of the peeled pin was clean, without any residues. On the other hand, after delamination, islands of the rubber are left on the every pin, which was fixed at the $\mathrm{Zr}$ modified rubber plates. Obviously there is no direct correlation of the degree of interface mixing, e.g. Me content in the rubber and adhesion. For example, the authors of [10] consider that interfacial layers formed during the moulding have a thickness that depends on the compound, but $6 \mathrm{~nm}$ can be taken as an indicative figure. Indeed, reactions accompanying SIAD process are very complex and complicated, involving substitution, replacement, interdiffusion, new phase formation, and so on. Their mechanisms are additionally interfered by oxidation, taking place simultaneously [10]. All this may make the surface layer of the coated rubber stiffer.

\section{Summary}

We have shown that Zr-based coatings produced by means of SIAD have complicated composition. Thin films deposited on rubber consist of Me, C, O, H. The SIAD of the Me-coatings causes strong modification of the macroscopic properties of the near surface region of the rubber. The SIAD process may successfully control the level of adhesion of the coating to the rubber.

\section{References}

[1] H. Fladry, N. Tengen, G.K. Wolf, Nucl. Instrum. Methods Phys. Res. B 91, 575 (1994). 
[2] V.S. Kulikauskas, I.S. Tashlykov, M.G. Shadrukhin, A.V. Kasperovich, Phys. Chem. Mech. Surf. 11, 965 (1995).

[3] I.S. Tashlykov, I.M. Belyi, Patent of Republic of Belarus, No. 2324

[4] I.S. Tashlykov, V.I. Kasperovich, M.G. Shadrukhin, A.V. Kasperovich, G.K. Wolf, W. Wesch, Surf. Coat. Technol. 116-119, 848 (1999).

[5] A.M. Mazurkevich, V.V. Chodasevich, V.V. Uglov, V.A. Kutsanov, A.G. Serov, V.V. Ponkratov, A.G. Kobjak, V.V. Bobkov, Vacuum Techn. Technol. 3, 18 (1991).

[6] L. Doolitle, Nucl. Instrum. Methods. Phys. Res. B 15, 227 (1986).
[7] G.K. Wolf, Surf. Coat. Technol. 43/44, 920 (1990).

[8] D. Schneider, Th. Schwarz, B. Schultrich, Thin Solid Films 219, 92 (1992).

[9] I.S. Tashlykov, A.V. Kasperovich, S. Kalbitzer, S. Fabian, G. Carter, in: Proc. 2nd Int. Symp. NEET 2001 - New Electrical and Electronic Technologies and Their Industrial Implementation, Kazimierz Dolny (Poland), Lublin Technical University, Lublin 2001, p. 72.

[10] L.A. Reeves, D. Kiroski, D.E. Packham, Surf. Interface Anal. 23, 299 (1995). 